\title{
PARAMETER ESTIMATION OF MACROSCOPIC TRAFFIC SIMULATION MODEL*
}

\author{
by Chumchoke NANTHAWICHIT ${ }^{* *}$, and Takashi NAKATSUJI**
}

\section{Introduction}

According to still growing traffic demand, the advanced traffic control concepts in intelligent vehicle-highway system may be more extensively implemented in the near future. Macroscopic traffic flow models, which are able to handle large size of road network systems with the fast simulation time, are likely to be necessary for the research and development of new surveillance and control techniques. Such models contain a set of parameters, which has to be estimated according to real traffic data. Since model parameters have significant effects on the performance of the simulation, they have to be identified carefully. In general, the identification procedure is formulated as a parameter optimization problem, which can be solved based on iterative comparison of model estimates with real traffic variables. Various techniques can be used for this purpose. However, the choice primarily depends on the nature of the parameters: If they are insensitive to traffic situation, a static approach can be used. If they possess non-linearity, a random search technique will be effective to reach the optimum value. If they are sensitive to traffic condition, a dynamic method should be adopted.

Studies concerning macroscopic model parameters had been done by some researchers, however, there were few study on the comparison of model parameters estimation techniques. $\mathrm{Cremer}^{2)}$ analysed the stability of model parameters for a small single road section using complex integral, and designed the ranges of the values of parameters. Cremer and Papageorgiou ${ }^{3)}$ used Box Complex technique to identify the model parameters and investigated the sensitivity of the model with respect to parameter changes. Nevertheless, the sensitivity of parameters with respect to traffic condition was not investigated. Cremer ${ }^{2)}$ also suggested the possibility that model parameters can be identified by treating them as state variables in Kalman filtering technique (KFT), but neither formulation nor numerical experiment was proposed.

This study focuses on the comparison of those methods concerning with the parameter estimation of a particular macroscopic model. Two methods were selected for comparison; which are Nonlinear Least Square technique (NLT) as a gradient basis, and Box technique (BCT) as a random search technique. Next, the effects of traffic condition on the model parameters estimation and the accuracy of model prediction were investigated. Finally, it was investigated whether the estimation of traffic states using KFT is effective.

\section{Macroscopic Traffic Flow Model}

\section{(1) Macroscopic Model}

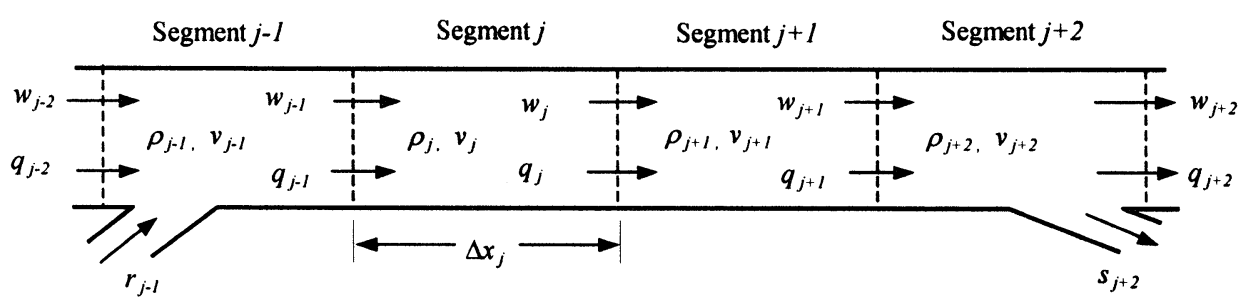

Fig. 1: Model of Freeway Section

In macroscopic model, discreatization in both time and space was adopted in the numerical processes to reduce the mathematical complexities. Fig. 1 shows space discreatization of a freeway section that consists of $J$ segments. Each segment is $\Delta x_{j}$ long. It is divided based on the assumption that traffic state (flow, density, and speed) is homogeneous within each segment. For stability consideration, to keep the solution within reasonable bounds, the time and the space increments $\Delta t, \Delta x$ must follow the rule that the time step, $\Delta t$, must be small enough and the space step, $\Delta x$, large enough so that vehicles cannot cross a full segment in one time step or $(\Delta x / \Delta t)$ should be more than the free flow speed ${ }^{14)}$. Macroscopic Traffic variables in the model were defined as follows:

$\rho_{j}(k)$ : density of segment $j$ at time $k$

$v_{j}(k)$ : space mean speed of segment $j$ at time $k$

$q_{j}(k)$ : flow rate at a point of boundary between segment $j$ and $j+1$ at time $k$

* Keywords: Parameter Identification, Macroscopic Model, FRESIM, Box Complex, Kalman Filtering

** Student Member of JSCE., Transportation and Traffic Systems, Graduate School of Engineering, Hokkaido University, (Kita-13, Nishi-8, Kita-ku, Sapporo, 060-8628, Fax:+81-11-706-6216)

*** Member of JSCE, Dr. Eng, Associate Prof., Transportation and Traffic Systems, Graduate School of Engineering, Hokkaido University, (Kita-13, Nishi-8, Kita-ku, Sapporo, 060-8628, Fax:+81-11-706-6216) 
$w_{j}(k)$ : time mean speed at a point of boundary between segment $j$ and $j+l$ at time $k$

$r_{j}(k)$ : ramp entry flow rate of segment $j$ at time $k$

$s_{j}(k)$ : ramp exit flow rate of segment $j$ at time $k$

Macroscopic traffic model, which was first derived by Payne ${ }^{12}$, is composed of a set of relationships among traffic volume, average traffic speed, and traffic density. Three relationships are required. The first relationship is fundamental relationship in traffic flow, volume, speed, and density:

$$
q_{j}(k)=\alpha\left(v_{j}(k) * \rho_{j}(k)\right)+(1-\alpha)\left(v_{j+1}(k) * \rho_{j+1}(k)\right)
$$

where $\quad k=$ time index;

$j=$ section index, and

$\alpha=$ weighting parameter ranging $0 \leq \alpha \leq 1$.

The above equation reflects that the volumes are determined at the edge of each segment, so the states of both neighboring segments may affect the actual flow. The second relationship is the continuity of vehicles that describes how density varies with time. It was pointed out by Lighthill and Whitham ${ }^{8)}$ as follow:

$$
\rho_{j}(k+1)=\rho_{j}(k)+\frac{\Delta t}{\Delta L_{j}}\left(q_{j-1}-q_{j}+r_{j}-s_{j}\right)_{(k)}
$$

where $\Delta t$ is time increment, and $\Delta L_{j}$ is length of $j^{\text {th }}$ section. The third relationship, which is so called the momentum equation, defines the variation of space mean speed over time. In this study, the version of Cremer and May ${ }^{4)}$, as the following, was adopted.

$$
v_{j}(k+1)=v_{j}(k)+\frac{\Delta t}{\tau}\left\{v_{e}\left[\rho_{j}(k)\right]-v_{j}(k)\right\}+\frac{\Delta t}{\Delta L_{j}} v_{j}(k)\left[v_{j-1}(k)-v_{j}(k)\right]-\frac{v \cdot \Delta t}{\tau \Delta L_{j}} \frac{\rho_{j+1}(k)-\rho_{j}(k)}{\rho_{j}(k)+\kappa}
$$

where $\tau=$ time constant,

$\kappa=$ density constant,

$v=$ anticipation constant, and

$v_{e}=$ speed at equilibrium state.

Model development and validation were shown in Cremer and May ${ }^{4)}$.

Presented in this paper is one of the so-called higher order continuum models, which is supposed to describe dynamic effects in traffic flow (i.e. acceleration and inertia effects of driver to adjust their speed according to traffic condition ahead, effect of driver to adjust their speed to equilibrium speed-density relationship, and effect of the propagation of speed difference). In Japan, mostly, a simple continuum model, where the mean speeds are taken directly from equilibrium speed-density relationship, was adopted. Deficiency in the simple macroscopic model can be referred in some literatures ${ }^{7)}{ }^{10}$, ${ }^{11}$. There are some works concerning with higher order models in Japan such as Pourmoallem, et.al. ${ }^{13)}$.

\section{(2) Equilibrium Speed-Density Relationship}

A relationship of speed and density at equilibrium state appearing in the second term on the right side of Equation 3, which is referred as to relaxation term, has a general expression proposed by May and $\mathrm{Keller}^{9)}$ as shown in Equation 4:

$$
v_{e}(\rho)=v_{f}\left[1-\left(\frac{\rho}{\rho_{j a m}}\right)^{a}\right]^{b},
$$

where $\rho_{\text {jam }}$ is the jam density, $v_{f i s}$ free-flow speed and $a, b$ are sensitivity factors which are positive numbers. In this study, the identification of these parameters was treated as another problem and estimated separately from the macroscopic parameters. The model parameters to be identified in this study are $\tau, v, \kappa$, and $\alpha$

\section{Traffic Data}

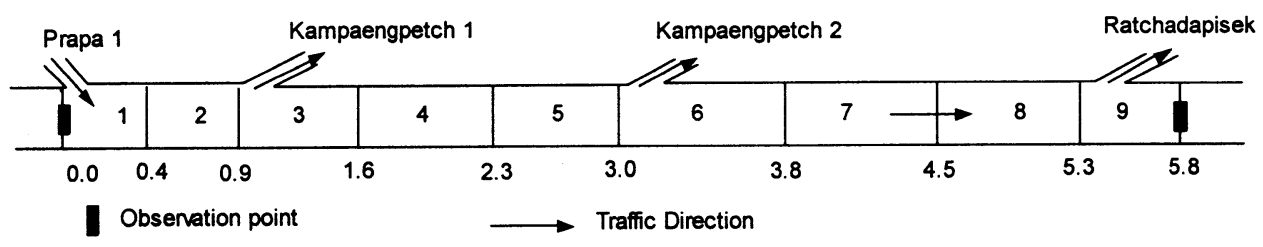

Fig. 2 Bangkok Site Study Road Section

Data from two sites are used in this study. For the first stage, this study used the data collected from outbound direction of the Second Stage Expressway of Bangkok, Thailand, between Vichaiyut Hospital and Kasemrat Hospital on Wednesday, December $3^{\text {rd }}, 1998$. The total length of study area is about 6 Kilometers. The traffic data at the entrance and the exit were observed by video camera, whereas the ramp data were observed manually. Two sets of data were collected during 14:00 to 15:30 hrs and 16:30 to 18:00hrs to cover the traffic situations of both off-peak and peak periods. These data are applied as cases 1 and 2 in numerical experiment. As shown in Fig. 2, the road section with three lanes was divided into 9 subsections ranging from 300 meters to 800 meters. The observation points for estimation programs are the entrance and exit of study road section. 
To obtain reliable model parameters being robust for various traffic conditions, the parameters have to be identified for extensive traffic situations. Hence the wide ranges of data from free-flow to congested conditions are required. It was almost impossible to obtain such data of Bangkok Expressway, in which no detector was installed, from actual fields. It requires vast efforts for data collection and compilation. In this study, the traffic data were generated by TRAF-FRESIM. The field data collected at the study area during a certain time period were used as input for FRESIM. The outflow volume, and spot speed at the exits were used to calibrate the influential parameters of FRESIM. The parameters calibrated are:

- Free-flow speed: $112 \mathrm{kph}$

- Parameter for collision avoidance time period: 1

- Minimum separation for generation of vehicles: 1.7 tenths of a second

Next the validation was performed so that the calibrated parameters were justified for the traffic data measured during another time period. The errors between FREESIM outputs and the real observation data were small enough for both peak and off-peak periods. After calibration and validation, extensive traffic data were produced using FRESIM by changing inflow volumes at entrances. Simulated data are used as case 3 in numerical experiment.

In order to examine the outcome of study at Bangkok, data from another site are applied in the second stage of study. The study data were selected from the 24-hr data observed by traffic detectors from Yokohane Line of Metropolitan Expressway in Tokyo, Japan, during February $20^{\text {th }}$ to $26^{\text {th }}$, 1996. The study road section is between Taishi Ramp and Namamugi Junction on the direction of heading to Tokyo. It is a 2-lane expressway with two on-ramp and one off-ramp. The length of study road section is about 7 Kilometers, which is divided into 12 subsections as depicted in Fig. 3. Although, detectors were installed in every subsection, the observation points applying in estimation programs are three points, which are the entrance and exit of study road section, and a point between subsection 5 and subsection 6 . The real observed data from Tokyo site are applied to the numerical experiment as data cases 4 , and 5 .

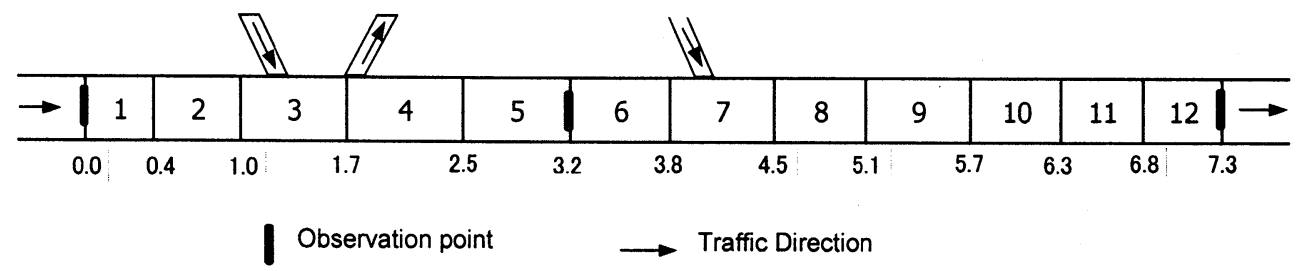

Fig. 3 Tokyo Site Study Road Section

\section{Parameter Estimation Technique}

\section{(1) Nonlinear Least Square Technique (NLT)}

The objective function, which is nonlinear equation, has to be transformed to be linear form using Taylor expansion technique. Once the equation becomes linear, the least square estimation technique can be applied. The objective function, J, was set as the error between observed variables and model outputs:

$$
J=\sum_{i=1}^{n}\left(\gamma_{q} \cdot\left(q_{i}-\hat{q}_{i}\right)^{2}+\gamma_{w} \cdot\left(w_{i}-\hat{w}_{i}\right)^{2}\right)
$$

To minimize the error, the differentiation of objective function with respect to model parameter is set to be zero, $\frac{\partial J}{\partial \beta_{m}}=0 \quad$, where $\beta_{m}$ is the model parameter of $\tau, \nu, \kappa$, and $\alpha$, and $\gamma_{q}, \gamma_{w}$ are the weighting factors of both volume and the speed errors. Normally, the reciprocals, $1 / \sigma_{q}{ }^{2}$ and $1 / \sigma_{w}{ }^{2}$, are used ${ }^{3)}$. They are chosen to be $10^{-5}$ and $10^{-2}$ respectively. The objective function is nonlinear with respect to the model parameters. By applying the Taylor Expansion to the objective function around the initial value of parameters and neglecting higher order term, model parameters can be estimated as:

$$
\begin{gathered}
\frac{\partial J}{\partial \hat{\beta}}=\left.\frac{\partial J}{\partial \hat{\beta}}\right|_{\hat{\beta}_{0}}+\frac{\partial^{2} J}{\partial \hat{\beta}_{1}^{2}}\left(\hat{\beta}_{1}-\hat{\beta}_{10}\right)+\ldots=0 \\
\hat{\beta}=\hat{\beta}_{0}-\left[\nabla^{2} \hat{\beta}_{0}\right]^{-1} \nabla \hat{\beta}_{0} \text { or } \\
{\left[\begin{array}{c}
\beta_{1} \\
\beta_{2} \\
\beta_{3} \\
: \\
\beta_{p}
\end{array}\right]=\left[\begin{array}{c}
\beta_{10} \\
\beta_{20} \\
\beta_{30} \\
\beta_{p 0}
\end{array}\right]-\left[\begin{array}{cccc}
\mathrm{S}_{11} & \mathrm{~S}_{12} & \ldots & \mathrm{S}_{1 \mathrm{p}} \\
\mathrm{S}_{21} & \mathrm{~S}_{22} & \\
: & & \ddots & \\
: & & \\
\mathrm{S}_{\mathrm{pl}} & \cdots & \cdots & \\
\end{array}\right] *\left[\begin{array}{c}
\mathrm{s}_{1 \mathrm{y}} \\
\mathrm{s}_{2 \mathrm{y}} \\
\vdots \\
: \\
\mathrm{s}_{\mathrm{py}}
\end{array}\right]}
\end{gathered}
$$

where $_{s_{m y}}=\frac{\partial J}{\partial \hat{\beta}_{m}}=\sum_{i=1}^{n}\left(-2 \gamma_{q} \cdot\left(q_{i}-\hat{q}_{i}\right) \cdot \frac{\partial q_{i}}{\partial \beta_{m}}-2 \gamma_{w} \cdot\left(w_{i}-\hat{w}_{i}\right) \cdot \frac{\partial w_{i}}{\partial \beta_{m}}\right)$ 


$$
S_{m l}=\frac{\partial^{2} J}{\partial \hat{\beta}_{m} \partial \beta_{l}}=\sum_{i=1}^{n}\left(\begin{array}{l}
2 \gamma_{q} \cdot\left(\frac{\partial q_{i}}{\partial \beta_{m}} \cdot \frac{\partial q_{i}}{\partial \beta_{l}}-\left(q_{i}-\hat{q}_{i}\right) \cdot \frac{\partial^{2} q_{i}}{\partial \beta_{m} \partial \beta_{l}}\right) \\
+2 \gamma_{w} \cdot\left(\frac{\partial w_{i}}{\partial \beta_{m}} \cdot \frac{\partial w_{i}}{\partial \beta_{l}}-\left(w_{i}-\hat{w}_{i}\right) \cdot \frac{\partial^{2} w_{i}}{\partial \beta_{m} \partial \beta_{l}}\right)
\end{array}\right)
$$

$i$ represents observation data at each time step and $m, l$ indicate the individual unknown parameters. Iterations are repeated until the changes of unknown parameters are small enough or no more improvement in the correlation between model variables.

\section{(2) Box Complex Technique (BCT)}

This method is a random search technique, which has proven effective in solving problem with nonlinear objective function subject to non-linear inequality constraints. The procedure should tend to find the global maximum due to the fact that the initial set of points is randomly scattered throughout the feasible region ${ }^{6}$. Unlike NLT, Box Complex algorithm does not require any derivatives. First, a number of complex points, which are the sets of model parameters, were generated randomly. The points must satisfy both explicit and implicit constraints. In this study, the explicit constraints are the ranges of the model parameters, while the maximum and minimum bounds of traffic speed and volume are the implicit constraints. If the explicit constraints are violated, the point is moved a small distance inside the violated limit. If an implicit constraint is violated, the point is moved one half of the distance to the centroid of the remaining points. After that, the objective function is evaluated at each point. The point having the highest function value is replaced by a point reflected through centroid of remaining points as the following equation.

$$
X_{i}(\text { new })=\delta\left(\bar{X}-X_{i}(\text { old })\right)+\bar{X}
$$

A value of $\delta=1.3$ is recommended by Box ${ }^{1)}$. A point that repeats in giving the highest function value on consecutive trials is moved one half the distance to the centroid of the remaining points. Iteration repeats until the objective function values of each point are nearly equal. To decide whether the global optimum is reached and to investigate the effect of initial value on this technique, the repetitions of the procedure while changing initial values were conducted.

Because of BCT have been used in the field of traffic engineering for a long time ${ }^{3), 11}$, it is applied in this study too. Other random search techniques such as genetic algorithm could be compared for the further study.

\section{Traffic State Estimation by Kalman Filter Technique (KFT)}

KFT can be described as a method for combining measurement data, which are contaminated with noise. In this technique, the estimated state is updated based on the observation data. It has been widely used in different fields such as automatic control system, mechanical, chemical processes, telecommunication, biology, nuclear reactors, and aeronautical engineering, etc. It can be applied to variety types of problems including state estimation ${ }^{5}$.

The state variables, $x_{k}$, compose of traffic density and space mean speed, while observation variable , $\mathrm{y}_{\mathrm{k}}$, are flow rate and time mean speed at each observation point. First, in KFT formulation, Equations. 2 and 3 were treated as state equations, while Equation. 1 was treated as the observation equation along with the following equation ${ }^{2}$ :

$$
w_{j}(k)=\alpha v_{j}(k)+(1-\alpha) v_{j+1}(k)
$$

where parameter $\alpha$ is the same as Equation 1. In addition, the white noise errors were induced in both macroscopic model formula and measurement process. Thus, the the state equation and observation equation become as follows:

$$
\begin{gathered}
\mathrm{x}(k+1)=\mathrm{f}[\mathrm{x}(k)]+\Gamma \varphi(k) \\
\mathrm{y}(k)=\mathrm{g}[\mathrm{x}(k)]+\psi(k)
\end{gathered}
$$

where

$$
\begin{array}{ll}
\Phi \text { is the error covariance matrix of state equations; } & \Phi=\mathrm{E}\left(\varphi(\mathrm{k}) \varphi^{T}(k)\right), \\
\Psi \text { is the error covariance matrix of observation equations; } & \Psi=\mathrm{E}\left(\psi(\mathrm{k}) \psi^{T}(k)\right),
\end{array}
$$

$$
\begin{aligned}
& \mathrm{x}(k)=\left(\rho_{1}, v_{1}, \ldots, \rho_{i}, v_{i}, \ldots \rho_{n}, v_{n}\right)_{(k)}^{T}, \\
& \mathrm{y}(k)=\left(q_{m 1}, w_{m 1}, \ldots, q_{m i}, w_{m i}, \ldots, q_{m n}, w_{m n}\right)_{(k)}^{T}, \\
& \varphi(k)=\left(\varphi_{1}^{\rho}, \varphi_{1}^{v}, \ldots, \varphi_{i}^{\rho}, \varphi_{i}^{v}, \ldots ., \varphi_{n}^{\rho}, \varphi_{n}^{\nu}\right)_{(k)}^{T}, \\
& \psi(k)=\left(\psi_{m 1}^{q}, \psi_{m 1}^{w}, \ldots ., \psi_{m i}^{q}, \psi_{m i}^{w}, \ldots ., \psi_{m n}^{q}, \psi_{m n}^{w}\right)_{(k)}^{T}, \\
& \Gamma=\operatorname{diag} \cdot\left(\frac{\Delta t}{\Delta L_{1}}, 1, \ldots ., \frac{\Delta t}{\Delta L_{i}}, 1, \ldots ., \frac{\Delta t}{\Delta L_{n}}, 1\right)_{(k)}^{T} .
\end{aligned}
$$

$i$ indicates each road segment $(i=1$ to $n$ ), whereas $m i$ is subscripted to specify the observation point number $(m i=m 1$ to $m n)$. In case that the entrance and exit points of study road section are observed the noises can be assigned as
$\varphi_{1}^{\rho}(k)=-\xi_{1}(k)$,
$\varphi_{n}^{\rho}(k)=\xi_{n-1}(k)$,
$\varphi_{i}^{v}(k)=\eta_{i}(k)$,
$\varphi_{i}^{\rho}(k)=\xi_{i-1}(k)-\xi_{i}(k)$ for the points that the data are not observed,
$\varphi_{i}^{\rho}(k)=\xi_{i-1}(k)$ for the observation points,
$\psi_{m i}^{q}(k)=\xi_{m i}(k)+\zeta_{q m i}(k), \quad \psi_{m i}^{w}(k)=\zeta_{w m i}(k)$, 
where $\varphi(k)$ and $\psi(k)$ are referred as modeling errors and measurement errors, respectively. $\xi$ is the system noise of volume estimation, $\eta$ is the system noise of speed estimation, $\zeta_{q}$ is the volume measurement error, and $\zeta_{w}$ is the speed measurement error. Finally linearize the state and observation equation around the nominal solution, $\widetilde{x}(k)$ using Taylor's expansion.

$$
\begin{gathered}
\widetilde{\mathrm{x}}(k+1) \cong \mathrm{f}[\hat{\mathrm{x}}(k)]+\frac{\partial \mathrm{f}}{\partial \mathrm{x}}(\mathrm{x}(\mathrm{k})-\hat{\mathrm{x}}(k))+\Gamma \varphi(k) \cong \mathrm{A}(k) \mathrm{x}(k)+\mathrm{b}(k)+\Gamma \varphi(k) \\
\widetilde{\mathrm{y}}(k) \cong \mathrm{g}[\mathrm{x}(k)]+\frac{\partial \mathrm{g}}{\partial \mathrm{x}}(\mathrm{x}(\mathrm{k})-\widetilde{\mathrm{x}}(k))+\psi(k) \cong \mathrm{C}(k) \mathrm{x}(k)+\mathrm{d}(k)+\psi(k)
\end{gathered}
$$

where

$$
\mathrm{b}(k)=\mathrm{f}[\hat{\mathrm{x}}(k)]-\frac{\partial \mathrm{f}}{\partial \mathrm{x}} \hat{\mathrm{x}}(k), \quad \mathrm{d}(k)=\mathrm{g}[\widetilde{\mathrm{x}}(k)]-\frac{\partial \mathrm{g}}{\partial \mathrm{x}} \widetilde{\mathrm{x}}(k),
$$

$$
\begin{aligned}
& \mathrm{A}(k)=\frac{\partial \mathrm{f}}{\partial \mathrm{x}}=\left[\frac{\partial\left(\rho_{1}, v_{1}, \ldots, \rho_{i}, v_{i}, \ldots, \rho_{n}, v_{n}\right)_{(k+1)}}{\partial\left(\rho_{1}, v_{1}, \ldots, \rho_{i}, v_{i}, \ldots, \rho_{n}, v_{n}\right)_{(k)}}\right]_{(2 \times n) \times(2 \times n)}, \\
& \mathrm{C}(k)=\frac{\partial \mathrm{g}}{\partial \mathrm{x}}=\left[\frac{\partial\left(q_{m 1}, w_{m 1}, \ldots, q_{m i}, w_{m i}, \ldots, q_{m n}, w_{m n}\right)_{(k)}}{\partial\left(\rho_{1}, v_{1}, \ldots, \rho_{i}, v_{i}, \ldots, \rho_{n}, v_{n}\right)_{(k)}}\right]_{(2 \times m n) \times(2 \times n)} .
\end{aligned}
$$

$\widetilde{\mathbf{x}}(k)$ is the estimated state vector before observing new data, $\mathbf{y}(k), \hat{\mathbf{x}}(k)$ is the updated vector after obtaining actaul measurement variables, $\mathbf{y}(k)$. By following the Kalman filtering algorithm step by step, the state variables can be corrected.

$$
\begin{aligned}
& \text { Step 1: } \widetilde{\mathrm{x}}(k)=\mathrm{f}[\hat{\mathrm{x}}(k-1)] \\
& \text { Step 2: } \mathrm{M}(k)=\mathrm{A}(k-1) \mathrm{P}(k-1) \mathrm{A}^{T}(k-1)+\Gamma \Phi \Gamma^{T} \\
& \text { Step 3: } \mathrm{K}(k)=\mathrm{M}(k) \mathrm{C}^{T}(k)\left[\mathrm{C}(k) \mathrm{M}(k) \mathrm{C}^{T}(k)+\Psi\right]^{-1} \\
& \text { Step 4: } \widetilde{\mathrm{y}}(k)=\mathrm{g}[\widetilde{\mathrm{x}}(k)] \\
& \text { Step 5: } \hat{\mathrm{x}}(k)=\widetilde{\mathrm{x}}(k)+\mathrm{K}(k)[\mathrm{y}(k)-\widetilde{\mathrm{y}}(k)] \\
& \text { Step 6: } \mathrm{P}(k)=\mathrm{M}(k)-\mathrm{K}(k) \mathrm{C}(k) \mathrm{M}(k) \\
& \text { Step 7: } k=k+1 \text {,go back to step 1 untill the required time step is reached. }
\end{aligned}
$$

\section{Numerical Experiments}

The mainstream volume $q_{0}(k)$ entering the section at the upstream end, on- and off-ramp volumes, the initial conditions of traffic situation, and the initial values of parameters were taken as inputs into each developed parameter estimation program. The volume and spot speed at other measurement points were treated as output variables to be compared to the corresponding model outputs. The obtained parameters then were applied to the macroscopic model to simulate the traffic situations. Simulation interval using in study is 10 seconds, while the road segment lengths are between 300 to 800 meters. So the $\Delta x / \Delta t<$ free flow speed satisfying the stability rule as mentioned above. Various sets of data covering a large variety of different traffic situations were run for result comparison. For Bangkok data, three cases are examined:

Case 1: Off-peak period: traffic data observed during 14:10 to 14:30 hrs.

Case 2: Peak period: traffic data observed during 16:40 to 17:00 hrs.

Case 3: Generated data by FRESIM, for comparison propose, with inflow volume between 4800 to $5200 \mathrm{vph}$, in this case traffic situation is not so fluctuant.

Two cases of traffic conditions from Tokyo data are:

Case 4: data on Feb 21th, Wed. between 16:00 to 16:30 hrs, low density traffic and quite smooth traffic.

Case 5: data on Feb 23th, Fri. between 06:30 to 07:00 hrs, traffic density changed rapidly from off-peak to morning peak.

The results by simulation runs of macroscopic model with a certain parameter sets estimated by each technique were compared with the real data. As the statistics principle, in order to evaluate each method quantitatively, the objective function J and root mean square of error (RMSE) of speed and volume were calculated.

\section{(1) Comparison between NLS and BCT}

At the first stage of study, Bangkok data were used. $\tau$ and $v$ were constrained in the range from 0 to $9999, \kappa$ was from 0 to 200 , and $\alpha$ was from 0 to 1 , respectively. For NLT, the model parameters were estimated with changing the initial values randomly of 50 sets. In BCT, three sets of initial parameters shown in Tables 1 were first applied. They are taken from Cremer and Papageorgiou ${ }^{3)}$, Papageorgiou, et al. ${ }^{11)}$, and Cremer and $\mathrm{May}^{4)}$, respectively. Furthermore, different numbers of complex points, which yielded 10, 25, and 50 sets of initial values, were also examined. The estimated parameters for each case are summarized in Tables 2. Fig. 4 illustrates the example of

Table 1 Initial Value for BCT

\begin{tabular}{|c|c|c|c|c|}
\hline No. & $\tau$ & $v$ & $\kappa$ & $\alpha$ \\
\hline 1 & 34.0 & 21.6 & 20.0 & 0.80 \\
2 & 72.0 & 28.0 & 40.0 & 0.80 \\
3 & 20.4 & 23.9 & 28.5 & 0.95 \\
\hline
\end{tabular}
parameter estimation output from NLT and BCT. It shows the estimation of $v$ for case 1 by changing the initial values and constraints of parameters.

Experimental results indicate that, in NLT, different initial values often resulted in absolutely different solutions for all three cases. In other words, the parameters strongly depend on the initial values. Furthermore, little improvement was gained even if 
the program started with different initial values. The possible reason is that the objective function is nonlinear and has a lot of extreme values. Due to the nature of NLT based on derivatives, it is very difficult to escape from a local minimum once entrapped.

As shown in Table 2, BCT produced better estimates for all 3 indices, including objective function (J), RMSE of volume and spot speed, than NLT in all cases. The initial values had small effect on the final solutions because BCT has such a mechanism that generates a number of random points automatically with avoiding a local minimum. Consequently, the method successfully yielded the parameters that were substantially different from the initial values. Moreover, the calculation process of BCT is much simpler than NLT because it does not require any derivative and matrix operations as NLT. In addition, Table 2 indicates that even BCT was effective in estimating the parameters for the off-peak of Case 1 and smooth traffic state of Case 3, but the error indices from model prediction are still large for case 2. By comparing three cases, the model with estimated parameter from both techniques can predict the traffic condition most accurately for Case 3 (quite smooth traffic state) and produces the worst outcome for case 2 (high volume and fluctuated traffic state). This feature reflects the efficiency of the macroscopic model itself.

Table 2 Parameter Estimated by NLT and BCT for Bangkok Data

\begin{tabular}{|c|c|c|c|c|c|c|}
\hline \multirow{2}{*}{ Technique } & \multicolumn{2}{|c|}{ Case 1 } & \multicolumn{2}{c|}{ Case 2 } & \multicolumn{2}{c|}{ Case 3 } \\
\cline { 2 - 7 } & NLT & BCT & NLT & BCT & NLT & BCT \\
\hline Initial Points & 50 & 50 & 50 & 50 & 50 & 50 \\
\hline$\tau(\mathrm{sec})$ & 20.41 & 8.12 & 518.47 & 242.51 & 301.26 & 419.93 \\
\hline$v\left(\mathrm{~km}^{2} / \mathrm{hr}\right)$ & 189.41 & 19.53 & 1565.40 & 630.12 & 1662.08 & 740.00 \\
\hline$\kappa(\mathrm{vkp})$ & 200.0 & 17.3 & 200.0 & 150.9 & 200.0 & 13.9 \\
\hline$\alpha$ & 1.000 & 0.888 & 0.961 & 1.000 & 0.963 & 0.989 \\
\hline $\begin{array}{c}\text { J per number } \\
\text { of observed data }\end{array}$ & 0.252 & 0.231 & 0.640 & 0.584 & 0.019 & 0.014 \\
\hline RMSE $_{\mathrm{q}}(\mathrm{vph})$ & 323.23 & 312.51 & 572.07 & 544.04 & 68.18 & 45.27 \\
\hline $\begin{array}{c}\text { RMSE } \\
\text { R }(\mathrm{kph})\end{array}$ & 3.85 & 3.65 & 5.59 & 5.37 & 1.29 & 1.15 \\
\hline
\end{tabular}

To investigate the above aspects more concretely, the experiments were extended to the data from expressway in Tokyo. Data from Tokyo site have different characteristics from Bangkok data, including road condition, number of lanes, speed limit, and driver behavior, etc. Moreover, data from Tokyo site are collected by traffic detectors installed in every segment. So it is possible to increase the number measurement points in each estimation process. Also detector data are possible to be converted to space mean speed and traffic density for comparison purpose.

As in the first stage, it mainly aimed to find the actual optimum set of parameters by each technique, so the ranges of parameters used in the first stage are very wide. As a result, some estimated values of parameters might not be practical to adopt in real world case even they are the global optimum. In the second stage, the ranges or constraints of parameters are more confining, e.g. $0-100$ for $\tau$ and $v$, and $0-1$ for $\alpha$. $\kappa$ was not taken into the estimation in this stage. It was set to be $40 \mathrm{vpk}^{11)}$. The accuracy of model should be compensated by the proper value of $v$, which appears in the same term of momentum equation. Besides, the model output is not so sensitive to the value of $\kappa$ (by sensitivity analysis, the most explicitly sensitive parameter to the model performance is $\alpha$, as it is only one parameter in observation equation).

The results of parameter estimation for Tokyo data (case 4, and case 5) by NLT and BCT are summarized in Table 3. The results show the similar trend as Bangkok data, e.g. BCT gives more consistent output while changing the initial value, and more precise optimum point can be obtained from BCT. However, the results indicate that by narrowing down the constraints of parameters, NLT can give almost same result as BCT. While in the large space of possible parameter's value as applied in first stage of study, BCT obviously yields the better result. In addition, as same as Bangkok data, the model prediction by optimum parameters was justified for the quite smooth traffic case (Case 4) but it was not justified for the traffic data that includes the rapid transient period (Case 5).

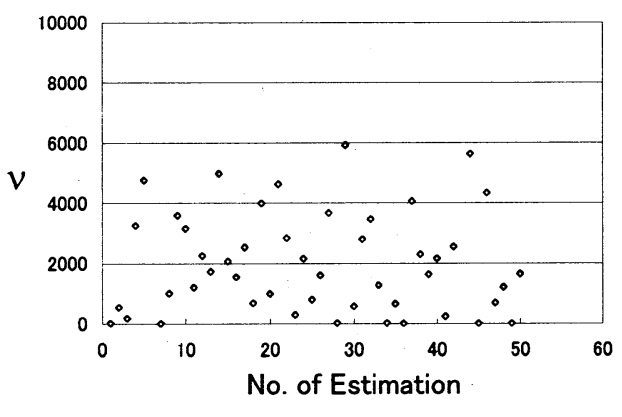

NLT-Estimation

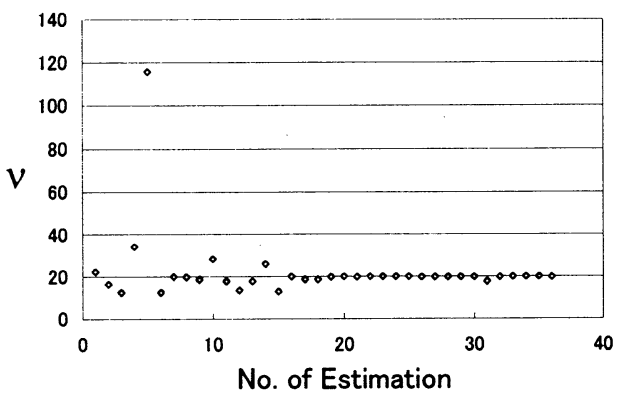

BCT-Estimation

Fig. 4 Comparison between NLT and BCT Estimation by Changing the Initial Value and Constraints

Table.3 Parameter Estimation by NLT and BCT for Tokyo Data

\begin{tabular}{|c|r|r|r|r|}
\hline \multirow{2}{*}{ Technique } & \multicolumn{2}{|c|}{ Case 4} & \multicolumn{2}{c|}{ Case 5 } \\
\cline { 2 - 5 } & NLT & \multicolumn{1}{c|}{ BCT } & NLT & \multicolumn{1}{c|}{ BCT } \\
\hline Initial Points & \multicolumn{1}{c|}{50} & 50 & 50 & \multicolumn{1}{c|}{50} \\
\hline$\tau(\mathrm{sec})$ & 82.62 & 64.08 & 57.06 & 54.66 \\
\hline$v\left(\mathrm{~km}^{2} / \mathrm{hr}\right)$ & 61.86 & 64.80 & 14.15 & 13.32 \\
\hline$\alpha$ & 0.954 & 0.924 & 0.614 & 1.000 \\
\hline $\begin{array}{c}\mathrm{J} \text { per number } \\
\text { of observed data }\end{array}$ & 0.382 & 0.381 & 1.172 & 0.945 \\
\hline RMSE $_{\mathrm{q}}(\mathrm{vph})$ & 143.34 & 143.10 & 240.31 & 226.36 \\
\hline $\mathrm{RMSE}_{\mathrm{w}}(\mathrm{kph})$ & 4.21 & 4.32 & 7.73 & 6.64 \\
\hline
\end{tabular}




\section{(2) Effect of Traffic Condition}

So far, the estimation is not successful in Case 2 and Case 5 as the error indices are still large comparing to other cases. Fig.5 shows the variation of spot speed observed at entrance and exit points as well as those predicted by the macroscopic model with the parameters estimated by BCT for Case 2. Although the estimated speed and volume approximately follow observed one on the average, the difference in the short term is still large. As shown in Fig. 5, there was a sudden speed drop around 16:50. In other words, traffic situations became congested after the time point. To treat this phenomenon more precisely, the data set of Case 2 and Case 5 were divided into two parts; before and after the abrupt change of speed. Then the parameters were identified separately by BCT. Table 4 exhibits the new parameters for each time period. With being aggregated for both periods, the separation was effective in improving both the objective function and the RMSE of spot speed. Nevertheless, the RMSE of both volume and speed are still large compared to the other cases. Further improvement is required. So it suggests that parameters should be varying in time according to traffic condition. Or else, the real-time prediction should be applied to state estimation problem.

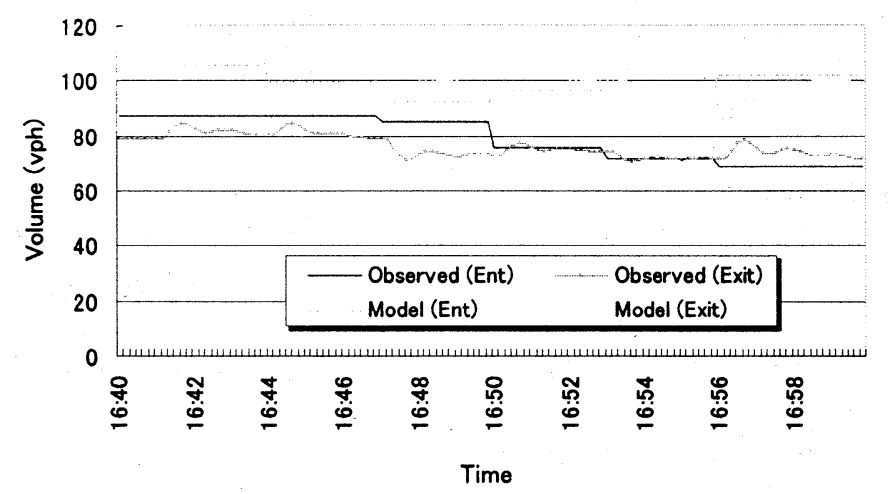

Fig. 5 Spot Speed Estimated by Optimum Parameters using BCT for Case 2

\section{(3) Real Time Estimation of Traffic States using} Kalman Filtering Technique

Up to now, model parameters were identified so that the different between model estimated and observed data were minimized. The traffic volume and speed were simplistically estimated by the macroscopic model. There was no adjustment by the observed data. Because of the inherence of the macroscopic model, traffic states cannot be properly identified by the model with lumped parameters in the case that traffic condition changes rapidly. That is, the real time estimation may work well in the ultimate sense. The KFT, which had been proven to be effective in state estimation problem in many fields, was used in this study as a real-time state estimation technique based on the feedback information. As shown in Tables 2 and 3, the data of Case 2 and Case 5 , which still have large errors while using the optimum parameters, were selected for this study. The traffic states first estimated by the macroscopic model were adjusted by the observed data using KFT with minimizing the difference in traffic volume. Table 5 shows the values of objective function and the errors of time mean speed estimated by the macroscopic model with KFT comparing to those estimated by the macroscopic model without KFT. It indicates that KFT improved the estimation precision for both Case 2 and Case 5.

\section{Conclusion}

Accuracy of traffic state estimation of macroscopic traffic flow model strongly depends on the model parameter. Thus parameter estimation problem plays a vital role in traffic state estimation. Efficiency of two techniques was compared in this study using various sets of data from the two different locations. The BCT can

Table 5 Comparison of Performance Indices between Simple Macroscopic Model and Macroscopic Model with KFT

\begin{tabular}{|c|c|c|c|c|}
\hline & \multicolumn{2}{|c|}{$\begin{array}{c}\text { J per number of } \\
\text { observation data }\end{array}$} & \multicolumn{2}{|c|}{ RMSE $_{\mathrm{w}}$} \\
\hline Case & Case 2 & Case 5 & Case 2 & Case 5 \\
\hline $\begin{array}{c}\text { Macroscopic Model } \\
\text { without KFT }\end{array}$ & 0.584 & 0.945 & 5.37 & 6.64 \\
\hline $\begin{array}{c}\text { Macroscopic Model } \\
\text { with KFT }\end{array}$ & 0.183 & 0.107 & 4.29 & 3.28 \\
\hline
\end{tabular}
be considered to be a superior technique comparing with the NLT in estimating the macroscopic model parameters. Its mechanism used to optimize the objective function with constraints is quite effective. It provides better results, and requires less computation effort than the NLT. The NLT fails to estimate the parameters if appropriate initial values are not adopted. However, NLT estimation can be improved if the ranges of parameters are more confining.

As a lumped estimation, BCT provides the superb outcomes in the cases that traffic volume is low or traffic condition gradually changes, but its performance deteriorates in the high volume with abrupt change in traffic condition. This means the model parameters strongly depend on the traffic condition. One could infer that the estimation can be improved if the model 
parameters are adjusted frequently according to traffic condition. Furthermore, some researchers, such as Michalopoulos, et. al ${ }^{10)}$, and Pourmoallem, et.al. ${ }^{13)}$, proposed the macroscopic models with some model parameters as functions of traffic states. Hence final goal should be simultaneous estimation of both traffic states and model parameters. The dynamic technique that possesses such ability, such as KFT, should be applied to this problem. Finally, as the macroscopic model cannot capture the real condition for some cases, especially in case of traffic condition changes rapidly, it was confirmed by this study that KFT, as a dynamic technique, is able to improve the traffic state estimation precision.

Result of study indicates that KFT can improve the model estimation. For that reason, if the model parameters are included into the state equation, KFT has a potential to simultaneously estimate model parameters as well as traffic state variables in real time manner. The state variables and model parameters are adjusted every time step so that the difference between estimated and observed measurement variables should be minimized. Consequently the traffic state prediction might be improved. However, the integration of model parameters into state variables requires another difficulty in formulating of the matrices $\mathrm{A}$ and $\mathrm{C}$ as well as inefficiencies in computation efforts. That will be the topic for further study

\section{References}

1) Box. M.J.: "A New Method of Constrained Optimization and a Comparison with Other Methods". Computer J., 8, pp. 42-52, 1965.

2) Cremer, M.: Der Verkehrsflu $\beta$ auf Schnellstraßen, Springer Verlag, New York, 1979.

3) Cremer, M. and Papageorgiou, M.: "Parameter Identification for a Traffic Flow Model". Automatica, Vol. 17, No. 6, pp. 837-843, 1981.

4) Cremer, M., and May, A.D.: "An Extended Traffic Flow Model For Inner Urban Freeways". Proc. $5^{\text {th }}$ IFAC/IFIP/IFORS Intern. Conf. On Control in Transportation Systems, Vienna, Austria, pp. 383-388, 1986.

5) Eykhoff, P.: System Identification: Parameter and State Estimation, John Wiley \& Sons, Great Britain, 1974.

6) Kuester, J. L. ,and Mize, J. H.: "Box (Complex Algorithm)". Optimization Techniques with Fortran, McGraw-Hill, Inc., New York, 1973.

7) Lebacque, J.P., and Lesort, J.B.: "Macroscopic Traffic Flow Models: A Question of Order". Proceedings of the 14 International Symposium on Transportation and Traffic Theory, Jerusalem, Israel, pp. 3-25, 1999.

8) Lighthill, M.H., and Witham, G.B.: "On Kinematic Waves II: A Theory of Traffic Flow on Long Crowded Roads". Proc., Royal Society, Series A229, No. 1178, London, England, pp. 317-345, 1971.

9) May, Jr.A.D., and Keller, H.E.M.: "Noninteger Car-Following Models". Highway Res. Rec. 199,pp. 19-32, 1967.

10) Michalopoulos, P.G., Yi, P., and Lyrintzis, A.S., Continuum Modelling of Traffic Dynamics for Congested Freeways, Transportation Research Part B, Vol. 27B, No. 4, pp. 315-332, 1993.

11) Papageorgiou, M., Blosseville, J.M., and Hadji-Salem, H.: "Macroscopic Modelling of Traffic Flow on the Boulevard Peripherique in Paris". Transportation Research Part B, Vol. 13B, No. 1, pp. 29-47, 1989.

12) Payne, H.J.: "Models of Freeway Traffic and Control". Simulation Council Proc., Mathematics of Public Systems, Vol. 1, No. 1, pp. 51-61,1971.

13) Pourmoallem, N., Nakatsuji, T., and Kawamura, A.: “A Neural-Kalman Filtering Method for Estimating Traffic States on Freeways". Journal of Infrastructure Planning and Management, No. 569/IV-36, pp. 105-114, 1997.

14) Ross, P.: Some Properties of Macroscopic Traffic Models". Transportation Research Record 1194, TRB, National Research Council, Washington, D.C., pp. 129-134, 1988.

\section{Parameter Estimation of Macroscopic Traffic Simulation Model}

by Chumchoke NANTHAWICHIT ${ }^{* *}$, and Takashi NAKATSUJI ${ }^{* * *}$ Ability of Macroscopic model parameter estimation by Nonlinear Least Square technique (NLT) and Box Complex technique (BCT) were compared using various conditions of traffic data from Bangkok and Tokyo. Experimental results indicate that BCT is superior to NLT in parameter estimation. Moreover it was found that the parameters strongly depend on traffic condition, or the parameters are varying with time. So the dynamic technique should be introduced to the parameter estimation problem. Finally, the prediction of traffic state by simple macroscopic model was compared with the real-time prediction based on feed back information using Kalman Filtering technique.

マクロ交通流モデルのパラメータ推定について

圧縮流体型のマクロ交通流モデルのパラメータ推定に関し、1）推定手法の比較、2）パラメータの交通状 態依存性、3）カルマン・フィルターによる交通状態推定の有効性に関する分析を行った。推定手法に関し ては、制約条件付の非線型最小自乗法とランダムサーチに基づくBox's Complex法の比較を行い、後者がほ とんどのケースにおいて有効であった。交通状態に応して時間带ことにパラメータを推定することによって 推定誤差を減少させることができる。また、Kalmanフィルターによって観測データに基づいて交通状態の推 定值をフィードバック的に補正することによってさらに推定の精度を高めることができる。 